\title{
An Implicit Algorithm for Finding a Fixed Point of a Q-Nonexpansive Mapping in Locally Convex Spaces
}

\author{
E. Soori $\mathbb{D}^{1},{ }^{1}$ M.R. Omidi, ${ }^{2}$ A.P. Farajzadeh $\mathbb{D},{ }^{3}$ and Yuanheng Wang $\mathbb{D}^{4}$ \\ ${ }^{1}$ Department of Mathematics, Lorestan University, P.O. Box 465, Khoramabad, Lorestan, Iran \\ ${ }^{2}$ Department of Basic Sciences, Kermanshah University of Technology, Kermanshah, Iran \\ ${ }^{3}$ Department of Mathematics, Razi University, Kermanshah, Iran \\ ${ }^{4}$ Department of Mathematics, Zhejiang Normal University, Jinhua, China
}

Correspondence should be addressed to Yuanheng Wang; yhwang@zjnu.cn

Received 26 March 2021; Accepted 29 July 2021; Published 13 August 2021

Academic Editor: Zoran Mitrovic

Copyright (c $2021 \mathrm{E}$. Soori et al. This is an open access article distributed under the Creative Commons Attribution License, which permits unrestricted use, distribution, and reproduction in any medium, provided the original work is properly cited.

In this paper, the notion of the $q$-duality mappings in locally convex spaces is introduced. An implicit method for finding a fixed point of a Q-nonexpansive mapping is provided. Finally, the convergence of the proposed implicit scheme is investigated. Some examples in order to illustrate of the main results are presented.

\section{Introduction}

Let $C$ be a nonempty closed and convex subset of a Banach space $E$ and $E^{*}$ be the dual space of $E$. Let $\langle.,$.$\rangle denote the$ pairing between $E$ and $E^{*}$. The normalized duality mapping $J: E \longrightarrow E^{*}$ is defined by

$$
J(x)=\left\{f \in E^{*}:\langle x, f\rangle=\|x\|^{2}=\|f\|^{2}\right\},
$$

for all $x \in E$. It is well known that if $J$ is a single-valued mapping, then it is norm-weak ${ }^{*}$ continuous (see, for more details, Theorem 2.6.10 in [1]). In this paper, the duality mapping of locally convex space is studied and denoted by $J_{q}$ for the seminorm $q$.

Suppose that $Q$ is a family of seminorms on a locally convex space $E$ which determines the topology of $E$ that will be denoted by $\tau_{Q}$. Let $C$ be a nonempty closed and convex subset of $E$. A mapping $T: C \longrightarrow C$ is called Q-nonexpansive if $q(T x-T y) \leq q(x-y)$, for all $x, y \in C$ and $q \in Q$. Also, the function $f: E \longrightarrow E$ is $Q$-contraction if there exists $\beta \in[0,1)$ such that $q(f(x)-f(y)) \leq \beta q(x-y)$, for all $x, y \in C$ and $q \in Q$.

The methods of iterations, which find a number of real applications, are popular for problems in sciences and engineering; see, e.g., [2-6]. Recently, various iterative algorithms have been investigated for fixed points of the nonlinear mapping, especially, for the mappings of nonexpansive type; see, e.g., [7-11] and the references therein.

Also, the general implicit algorithm for finding a fixed point of a $Q$-nonexpansive mapping is given by

$$
z_{n}=\varepsilon_{n} f z_{n}+\left(1-\varepsilon_{n}\right) T z_{n}, \quad(n=1,2, \ldots),
$$

where $f$ is a $Q$-contraction and $T$ is a $Q$-nonexpansive mapping. On the contrary, it is proven that there exists a sunny $Q$-nonexpansive retraction $P$ of $C$ onto $\operatorname{Fix}(T)$ and $x \in C$ such that the the sequence $\left\{z_{n}\right\}$ converges to $P x$ with respect to the topology $\tau_{Q}$.

Moreover, some notions and concepts in locally convex spaces are recalled. The Hahn-Banach theorem and Banach contraction principle will be generalized to locally convex spaces. The results of this article can be viewed as implicit and locally convex space version of some results given in $[1,12-17]$.

\section{Methods and Preliminaries}

Suppose that $Q$ is a family of seminorms on a locally convex space $E$ which determines the topology of $E$ will be denoted by $\tau_{Q}$. 
Let $D \subseteq B \subseteq E$ and $P$ be a retraction of $B$ onto $D$, that is, $P x=x$, for each $x \in D$. Then, $P$ is said to be sunny if, for each $x \in B$ and $t \geq 0$ with $P x+t(x-P x) \in B$, we have

$$
P(P x+t(x-P x))=P x
$$

A subset $D$ of $B$ is said to be sunny $Q$-nonexpansive retract of $B$ if there exists a sunny $Q$-nonexpansive retraction $P$ of $B$ onto $D$. We know that if $E$ is a smooth Banach space and $P$ a retraction of $B$ onto $D$, then $P$ is sunny nonexpansive if and only if

$$
\langle x-P x, J(z-P x)\rangle \leq 0,
$$

for each $x \in B$ and $z \in D$. For more details, see [18]. In the sequel, we will prove inequality (4) for a real locally convex space.

Now, we recall the following definitions:

(1) The locally convex topology $\tau_{Q}$ is separated if and only if the family of seminorms $Q$ possesses the following property: for each $x \in E \backslash\{0\}$, there exists $q \in Q$ such that $q(x) \neq 0$ (see [19]) or equivalently

$$
\bigcap_{q \in Q}\{x \in E: q(x)=0\}=\{0\} .
$$

(2) Let $E$ be a locally convex topological vector space over $\mathbb{R}$ or $\mathbb{C}$. If $U \subset E$, the polar of $U$ is denoted by

$$
U^{\circ}=\left\{f \in E^{*}:|f(x)| \leq 1, \forall x \in U\right\},
$$

see [20].

(3) Suppose $G$ is a Hausdorff topological group, and $\left(x_{\alpha}\right)$ is a net in $G$ defined on a directed set $D .\left(x_{\alpha}\right)$ is left Cauchy when the following happens: for every neighborhood $B$ of the identity $e$ of $G$, there exists an $\alpha_{0} \in D$ such that

$$
\begin{array}{r}
\forall \beta, \gamma \in D: \beta>\alpha_{0}, \\
\gamma>\alpha_{0} \Longrightarrow x_{\beta}^{-1} x_{\gamma} \in B,
\end{array}
$$

see [20].

Suppose that $Q$ is a family of seminorms on a locally convex space $X$ which determines the topology of $X$ and $q \in Q$. Let $Y$ be a subset of $X$ and

$$
q_{Y}^{*}(f)=\sup \{|f(y)|: y \in Y, q(y) \leq 1\} .
$$

Also,

$$
q^{*}(f)=\sup \{|f(x)|: x \in X, q(x) \leq 1\},
$$

for every linear functional $f$ on $X$. Notice that if, for each $x \in X, q(x) \neq 0$, and $f \in X^{*}$, then

$$
|\langle x, f\rangle| \leq q(x) q^{*}(f),
$$

for more details, see [12].

We will make use of the following theorems.
Theorem 1 (see [12]). Suppose that $Q$ is a family of seminorms on a real locally convex space $X$ which determines the topology of $X$ and $q \in Q$ is a continuous seminorm and $Y$ is a vector subspace of $X$ such that

$$
Y \cap\{x \in X: q(x)=0\}=\{0\} .
$$

Let $f$ be a real linear functional on $Y$ such that $q_{Y}^{*}(f)<\infty$. Then, there exists a continuous linear functional $h$ on $X$ that extends $f$ such that

$$
q_{Y}^{*}(f)=q^{*}(h)
$$

Theorem 2 (see [12]). Suppose that $Q$ is a family of seminorms on a real locally convex space $X$ which determines the topology of $X$ and $q \in Q$ a nonzero continuous seminorm. Let $x_{0}$ be a point in $X$. Then, there exists a continuous linear functional $f$ on $X$ such that $q^{*}(f)=1$ and $f\left(x_{0}\right)=q\left(x_{0}\right)$.

Next, we bring the following known results for easy reference.

Theorem 3 (see Proposition 2.5.2 in [1]). Let $X$ be a topological space and $f: X \longrightarrow(-\infty,+\infty]$ a function. Then, the following statements are equivalent: (a) $f$ is lower semicontinuous; (b) for each $\alpha \in \mathbb{R}$, the level set $\{x \in X: f(x) \leq \alpha\}$ is closed.

Theorem 4 (see Theorem 3.26 (Banach-Alaoglu) in [20]). Suppose that $X$ is a locally convex space and $U$ is a neighborhood of zero in $X$. Then, $U^{\circ}$, the polar set of $U$, is weak* compact.

For general topological spaces, we know the useful property as follows.

Theorem 5 (see Theorem 3.3.18 in [21]). For a topological space $(X, \tau)$, the following are equivalent:

(i) $X$ is compact

(ii) Each net in $X$ has a convergent subnet

\section{Main Result}

First, we define our notation of the $q$-duality mapping as follows.

Suppose that $Q$ is a family of seminorms on a real locally convex space $X$ which determines the topology of $X, q \in Q$ is a continuous seminorm, and $X^{*}$ is the dual space of $X$. A multivalued mapping $J_{q}: X \longrightarrow 2^{X^{*}}$, defined by

$$
J_{q} x=\left\{j \in X^{*}:\langle x, j\rangle=q(x)^{2}=q^{*}(j)^{2}\right\},
$$

is called $q$-duality mapping. Obviously, $J_{q}(-x)=-J_{q}(x)$ and $J_{q} x \neq \varnothing$. Indeed, let $x \in X$; if $q(x)=0$, then $j=0 \in J_{q} x$, and if $q(x) \neq 0$, by applying Theorem 2 , there exists a linear functional $f \in X^{*}$ such that $q^{*}(f)=1$ and $\langle x, f\rangle=q(x)$. Putting $j:=q(x) f$, then 


$$
\begin{aligned}
\langle x, j\rangle & =\langle x, q(x) f\rangle=q(x)\langle x, f\rangle=q(x)^{2}, \\
q^{*}(j) & =\sup \{|j(y)|: y \in X, q(y) \leq 1\} \\
& =\sup \{|q(x) f(y)|: y \in X, q(y) \leq 1\} \\
& =q(x) \sup \{|f(y)|: y \in X, q(y) \leq 1\} \\
& =q(x) q^{*}(f)=q(x),
\end{aligned}
$$

and then, $j=q(x) f \in J_{q} x$.

Now, we present the following result that extends Proposition 4.1.1 in [1].

Lemma 1. Let $Q$ be a family of seminorms on a Hausdorff and complete locally convex space $E$ which determines the topology of $E, \phi_{q}: E \longrightarrow(-\infty, \infty]$ a bounded below and lower semicontinuous function, for each $q \in Q$, and $\left\{x_{n}\right\}$ a sequence in $E$ such that $q\left(x_{n}-x_{n+1}\right) \leq \phi_{q}\left(x_{n}\right)-\phi_{q}\left(x_{n+1}\right)$, for all $n \in \mathbb{N}_{0}=\mathbb{N} \cup\{0\}$ and $q \in Q$. Then, $\left\{x_{n}\right\}$ converges to $a$ point $v \in E$, and for each $q \in Q$,

$$
q\left(x_{n}-v\right) \leq \phi_{q}\left(x_{n}\right)-\phi_{q}(v),
$$

for all $n \in \mathbb{N}_{0}$.

Proof. Since $q\left(x_{n}-x_{n+1}\right) \leq \phi_{q}\left(x_{n}\right)-\phi_{q}\left(x_{n+1}\right)$, for each $n \in \mathbb{N}_{0}$ and $q \in Q$, then $\left\{\phi_{q}\left(x_{n}\right)\right\}$ is a decreasing sequence for each $q \in \mathrm{Q}$. Moreover,

$$
\begin{aligned}
\sum_{n=0}^{m} q\left(x_{n}-x_{n+1}\right) & =q\left(x_{0}-x_{1}\right)+q\left(x_{1}-x_{2}\right)+\cdots+q\left(x_{m}-x_{m+1}\right) \\
& \leq \phi_{q}\left(x_{0}\right)-\phi_{q}\left(x_{m+1}\right) \leq \phi_{q}\left(x_{0}\right)-\inf _{n \in \mathbb{N}_{0}} \phi_{q}\left(x_{n}\right),
\end{aligned}
$$

for $m \in \mathbb{N}_{0}$. Letting $m \longrightarrow \infty$, we have

$$
\sum_{n=0}^{\infty} q\left(x_{n}-x_{n+1}\right)<\infty,
$$

and then, $\lim _{n} q\left(x_{n}-x_{n+1}\right)=0$, for each $q \in Q$. This implies that $\left\{x_{n}\right\}$ is a left Cauchy sequence in $E$. Since $E$ is Hausdorff and complete, there exists a unique $v \in E$ such that $\lim _{n \longrightarrow \infty} x_{n}=v$. Let $m, n \in \mathbb{N}_{0}$ with $m>n$. Then,

$$
\begin{aligned}
q\left(x_{n}-x_{m}\right) & \leq \sum_{i=n}^{m-1} q\left(x_{i}-x_{i+1}\right) \\
& \leq \phi_{q}\left(x_{n}\right)-\phi_{q}\left(x_{m}\right),
\end{aligned}
$$

for each $q \in Q$. Since $\phi_{q}$ is lower semicontinuous and $q \in Q$ is continuous (Theorem 1.4 in [19]), letting $m \longrightarrow \infty$, we conclude that

$$
q\left(x_{n}-v\right) \leq \phi_{q}\left(x_{n}\right)-\lim _{m \longrightarrow \infty} \phi_{q}\left(x_{m}\right) \leq \phi_{q}\left(x_{n}\right)-\phi_{q}(v),
$$

for all $n \in \mathbb{N}_{0}$ and $q \in Q$.

Now, we state an extension of Banach contraction principle to locally convex spaces, and we call it Banach Q-contraction principle.
Theorem 6 (Banach Q-contraction principle). Let $Q$ be a family of seminorms on a separated and complete locally convex space $E$ which determines the topology of $E$ and $T: E \longrightarrow E$ a Q-contraction mapping with Lipschitz constant $k \in(0,1)$. Then,

(a) Thas a unique fixed point $v \in E$.

(b) For arbitrary $x_{0} \in E$, the Picard iteration process defined by

$$
x_{n+1}=T\left(x_{n}\right), \quad n \in \mathbb{N}_{0},
$$

converges to $v$.

(c) $q\left(x_{n}-v\right) \leq\left(k^{n} /(1-k)\right) q\left(x_{0}-x_{1}\right)$, for all $n \in \mathbb{N}_{0}$ and $q \in Q$.

Proof

(a) For each $q \in Q$, let $\phi_{q}: E \longrightarrow \mathbb{R}^{+}$be a function defined by

$$
\phi_{q}(x)=\frac{q(x-T x)}{1-k},
$$

for each $x \in E$. Since $q$ is continuous, $\phi_{q}$ is also a continuous function. Also, $T$ is a Q-contraction mapping; then,

$$
q\left(T x-T^{2} x\right) \leq k q(x-T x),
$$

for each $x \in E$ and $q \in Q$, which conclude that

$$
q(x-T x)-k q(x-T x) \leq q(x-T x)-q\left(T x-T^{2} x\right) .
$$

Hence,

$$
\begin{aligned}
q(x-T x) & \leq \frac{1}{1-k}\left[q(x-T x)-q\left(T x-T^{2} x\right)\right] \\
& \leq \phi_{q}(x)-\phi_{q}(T x)
\end{aligned}
$$

for each $q \in Q$. Let $x$ be an arbitrary and fixed element in $E$. We define the sequence $x_{n}$ in $E$ by $x_{n}=$ $T^{n} x(n \in \mathbb{N})$. By using (24), we have

$$
q\left(x_{n}-x_{n+1}\right) \leq \phi_{q}\left(x_{n}\right)-\phi_{q}\left(x_{n+1}\right),
$$

for each $n \in \mathbb{N}$ and $q \in Q$. Since $E$ is Hausdorff, by applying Lemma 1, there exists an element $v \in E$ such that

$$
\begin{gathered}
\lim _{n \longrightarrow \infty} x_{n}=v, \\
q\left(x_{n}-v\right) \leq \phi_{q}\left(x_{n}\right),
\end{gathered}
$$

for each $n \in \mathbb{N}_{0}$ and $q \in Q$. Notice that $T$ is continuous (page 3 in [19]) and $x_{n+1}=T x_{n}$; therefore, $v=T v$. Let $z \in E$ be another fixed point for $T$ and $q(v-z)>0$ for some $q \in Q$. Then,

$0<q(v-z)=q(T v-T z) \leq k q(v-z)<q(v-z)$, 
which is a contradiction. Therefore, $q(v-z)=0$, for each $q \in Q$. Since $E$ is separated, we have $v=z$. Hence, $T$ has unique fixed point $v \in E$.

(b) This assertion follows from part (a).

(c) By applying (22), we have $\phi_{q}\left(x_{n}\right) \leq k^{n} \phi_{q}\left(x_{0}\right)$, for each $q \in Q$. This implies from (26) that $q\left(x_{n}-v\right) \leq k^{n} \phi_{q}\left(x_{0}\right)$, for each $q \in Q$.

Next, we prove a generalization of Proposition 2.4.7 in [1] as follows.

Lemma 2. Let $E$ be a locally convex space. Then, for $x, y \in E$ and for each $q \in Q$ with $q(x) \neq 0$, the following statements are equivalent:

(a) $q(x) \leq q(x+t y)$, for all $t>0$ with $q(x+t y) \neq 0$

(b) There exists $j_{q} \in J_{q} x$ such that $\left\langle y, j_{q}\right\rangle \geq 0$

\section{Proof}

(a) $\Longrightarrow$ (b). Let $t>0$ and $f_{t} \in J_{q}(x+t y)$, and we define $g_{t}=f_{t} / q^{*}\left(f_{t}\right)$. It is clear that $q^{*}\left(g_{t}\right)=1$ and $g_{t} \in\left(1 / q^{*}\left(f_{t}\right)\right) J_{q}(x+t y)$; then,

$$
\begin{aligned}
q(x) & \leq q(x+t y)=\frac{\left\langle x+t y, f_{t}\right\rangle}{q^{*}\left(f_{t}\right)} \\
& =\left\langle x+t y, g_{t}\right\rangle=\left\langle x, g_{t}\right\rangle+t\left\langle y, g_{t}\right\rangle \\
& \leq q(x)+t\left\langle y, g_{t}\right\rangle .
\end{aligned}
$$

Putting $U=\{x \in E: q(x) \leq 1\}$, it is clear that

$$
\left\{g_{t}\right\}_{t} \subset U^{\circ}=\left\{f \in E^{*}:|f(x)| \leq 1, \forall x \in U\right\},
$$

from Theorem $4, U^{\circ}$ is weak $^{*}$ compact; then, by applying Theorem 5, the net $\left\{g_{t}\right\}$ has a limit point $g \in E^{*}$ such that $q^{*}(g) \leq 1$. From the above equations $q(x) \leq\left\langle x+t y, g_{t}\right\rangle$, let $y=0$; by limiting, we have $q(x) \leq\langle x, g\rangle$. Also, since $t>0$, by using (28) and limiting, we have $\langle y, g\rangle \geq 0$. Therefore,

$$
q(x) \leq\langle x, g\rangle \leq q(x) q^{*}(g) \leq q(x)
$$

which conclude that $\langle x, g\rangle=q(x)$ and $q^{*}(g)=1$. Set $j_{q}=g q(x)$; then, $j_{q}(x)=g(x) q(x)=q(x)^{2}$ and

$$
\begin{aligned}
q^{*}\left(j_{q}\right) & =\sup \left\{\left|j_{q}(z)\right|: z \in X, q(z) \leq 1\right\} \\
& =\sup \{|q(x) g(z)|: z \in X, q(z) \leq 1\} \\
& =q(x) \sup \{|g(z)|: z \in X, q(z) \leq 1\} \\
& =q(x) q^{*}(g)=q(x) .
\end{aligned}
$$

Hence, $j_{q} \in J_{q} x$ and $\left\langle y, j_{q}\right\rangle=\langle y, g q(x)\rangle=q(x)$ $\langle y, g\rangle \geq 0$. (b) $\Longrightarrow$ (a). Suppose, for $x, y \in X$ and $q \in Q$ with $q(x) \neq 0$, there exists $j_{q} \in J_{q} x$ such that $\left\langle y, j_{q}\right\rangle \geq 0$. We know that

$$
\left|\left\langle x+t y, j_{q}\right\rangle\right| \leq q(x+t y) q^{*}\left(j_{q}\right)=q(x+t y) q(x) .
$$

Hence, for $t>0$ with $q(x+t y) \neq 0$, we have

$$
\begin{aligned}
q(x)^{2} & =\left\langle x, j_{q}\right\rangle \leq\left\langle x, j_{q}\right\rangle+\left\langle t y, j_{q}\right\rangle \\
& =\left\langle x+t y, j_{q}\right\rangle \leq q(x+t y) q(x)
\end{aligned}
$$

which implies that $q(x) \leq q(x+t y)$. This completes the proof.

The next theorem is significant in the sequel, and it extends Proposition 2.10.21 in [1].

Theorem 7. Let $E$ be a separated locally convex space, $C a$ nonempty convex subset of $E$, and $D$ a nonempty subset of $C$. Also, let $J_{q}: E \longrightarrow E^{*}$ be a single valued, for every $q \in Q$ and $P$ a retraction of $C$ onto $D$. Then, for each $q \in Q$, the following statements are equivalent:

(a) $\left\langle x-P x, J_{q}(y-P x)\right\rangle \leq 0,(x \in C, y \in D)$

(b) $P$ is sunny $Q-n o n e x p a n s i v e$

Proof

(a) $\Longrightarrow$ (b). First, we show that $P$ is sunny. For $x \in C$ and $t>0$, set $x_{t}:=P x+t(x-P x)$. Since $C$ is convex, $x_{t} \in C$, for each $t \in(0,1]$. Hence, from (a), we have

$$
\begin{aligned}
\left\langle x-P x, J_{q}\left(P x-P x_{t}\right)\right\rangle & \geq 0, \\
\left\langle x_{t}-P x_{t}, J_{q}\left(P x_{t}-P x\right)\right\rangle & \geq 0 .
\end{aligned}
$$

Because $x_{t}-P x=t(x-P x)$ and $\left\langle t(x-P x), J_{q}(P x\right.$ $\left.\left.-P x_{t}\right)\right\rangle \geq 0$, we have

$$
\left\langle x_{t}-P x, J_{q}\left(P x-P x_{t}\right)\right\rangle \geq 0 .
$$

Combining (34) and (35), we have

$$
\begin{aligned}
q\left(P x-P x_{t}\right)^{2}= & \left\langle P x-x_{t}+x_{t}-P x_{t}, J_{q}\left(P x-P x_{t}\right)\right\rangle \\
= & -\left\langle x_{t}-P x, J_{q}\left(P x-P x_{t}\right)\right\rangle \\
& +\left\langle x_{t}-P x_{t}, J_{q}\left(P x-P x_{t}\right)\right\rangle \leq 0 .
\end{aligned}
$$

Then, $q\left(P x-P x_{t}\right)=0$, for each $q \in Q$. Thus, from the fact that $E$ is separated, $P x=P x_{t}$. This means that $P$ is sunny. Now, we show that $P$ is $Q$-nonexpansive. For $x, z \in C$ and $q \in Q$, we have from (a) that $\left\langle x-P x, J_{q}(P x-P z)\right\rangle \geq 0$ and $\left\langle z-P z, J_{q}\right.$ $(P z-P x)\rangle \geq 0$. Hence, 


$$
\begin{aligned}
\left\langle x-z-(P x-P z), J_{q}(P x-P z)\right\rangle \geq & 0, \\
\left\langle x-z, J_{q}(P x-P z)\right\rangle= & \left\langle x-P x+P x-P z+P z-z, J_{q}(P x-P z)\right\rangle \\
= & \left\langle x-P x, J_{q}(P x-P z)\right\rangle-\left\langle z-P z, J_{q}(P x-P z)\right\rangle \\
& +\left\langle P x-P z, J_{q}(P x-P z)\right\rangle \text { geq }\left\langle P x-P z, J_{q}(P x-P z)\right\rangle \\
= & q^{2}(P x-P z) .
\end{aligned}
$$

(notice that $J_{q}(P x-P z)$ is single valued). On the contrary,

$$
\begin{aligned}
\left\langle x-z, J_{q}(P x-P z)\right\rangle & \leq q(x-z) q^{*}\left(J_{q}(P x-P z)\right) \\
& =q(x-z) q(P x-P z) .
\end{aligned}
$$

Therefore, be applying (37) and (38), we have

$$
q(P x-P z) \leq q(x-z)
$$

it follows that $P$ is $Q$-nonexpansive.

(b) $\Longrightarrow($ a). Conversely, suppose that $P$ is the sunny $Q$-nonexpansive retraction from $C$ onto $D, x \in C$ and $y \in D$. Then, $P x \in D$ and $P y=y$. Since $C$ is convex, $P x+t(x-P x) \in C$, for $0<t \leq 1$. Also, $P$ is sunny; then,

$$
P x=P(P x+t(x-P x))
$$

and therefore,

$$
\begin{aligned}
q(y-P x) & =q(P y-P x)=q(P y-P(P x+t(x-P x))) \\
& \leq q(y-(P x+t(x-P x))) \\
& =q(y-P x+t(P x-x)) .
\end{aligned}
$$
that

Hence, from Lemma 2, there exists $j_{q} \in J_{q}(y-P x)$ such

$$
\left\langle P x-x, j_{q}(y-P x)\right\rangle \geq 0, \quad x \in C, y \in D .
$$

Since $J_{q}: E \longrightarrow E^{*}$ is single valued, we can say that

$$
\left\langle x-P x, J_{q}(y-P x)\right\rangle \leq 0, \quad x \in C, y \in D .
$$

Next, as a direct consequence of Theorem 6.5.3 in [18], we have the following result. To apply Theorem 6.5.3 in [18] in Theorems 8, 9, 11, and 12 and Corollary 1, it is considered that inf $q(x)>0$, for each $q \in Q$, when $x$ is in $K(28)$ and (34), $C_{0}, \operatorname{Fix}(T)$, and $C$, respectively.

Theorem 8. Let $Q$ be a family of seminorms on a locally convex space $E$ which determines the topology of $E$ and $K$ be a convex subset of $E, x \in E$ and $x_{0} \in K$. Then, for each $q \in Q$, the following are equivalent:

(1) $q\left(x_{0}-x\right)=\inf \{q(x-y): y \in K\}$.
(2) There exists an $L_{q} \in E^{*}$ such that $L_{q}(y) \leq q(y)$, for each $y \in E, q^{*}\left(L_{q}\right)=1$, and

$$
\inf \left\{L_{q}(y-x): y \in K\right\}=q\left(x_{0}-x\right) \text {. }
$$

(3) There exists an $L_{q} \in E^{*}$ such that $L_{q}(y) \leq q(y)$, for each $y \in E, q^{*}\left(L_{q}\right)=1$, and

$$
\begin{aligned}
\inf \left\{L_{q}(y): y \in K\right\} & =L_{q}\left(x_{0}\right), \\
L_{q}\left(x_{0}-x\right) & =q\left(x_{0}-x\right) .
\end{aligned}
$$

The next theorem is similar to Corollary 6.5.5 in [18].

Theorem 9. Let $Q$ be a family of seminorms on a real locally convex space $E$ which determines the topology of $E$ and $K$ be a convex subset of $E, x \in E$ and $x_{0} \in K$. Then, for each $q \in Q$, the following statements are equivalent:

(1) $q\left(x_{0}-x\right)=\inf \{q(x-y): y \in K\}$

(2) There exists an $f_{q} \in J_{q}\left(x-x_{0}\right)$ such that $f_{q}\left(x_{0}-y\right) \geq 0$, for every $y \in K$

Proof

(1) $(1) \Longrightarrow(2)$. Let $q \in Q$ and $q\left(x_{0}-x\right)$ $=\inf \{q(x-y): y \in K\}$. Then, from Theorem 8, there exists an $L_{q} \in E^{*}$ such that $L_{q}(y) \leq q(y)$, for each $y \in E, q^{*}\left(L_{q}\right)=1$, and

$$
\inf \left\{L_{q}(y-x): y \in K\right\}=q\left(x_{0}-x\right) .
$$

Set $f_{q}=-q\left(x_{0}-x\right) L_{q}$. It is easy to see that $L_{q}\left(x_{0}-x\right)=q\left(x_{0}-x\right)$; therefore,

$$
\begin{aligned}
f_{q}\left(x-x_{0}\right) & =-q\left(x_{0}-x\right) L_{q}\left(x-x_{0}\right) \\
& =q^{2}\left(x_{0}-x\right)=\left(q^{*}\left(f_{q}\right)\right)^{2} .
\end{aligned}
$$

Then, $f_{q} \in J_{q}\left(x-x_{0}\right)$. Hence, from (46) and (47), we have

$$
\begin{aligned}
f_{q}\left(x_{0}-y\right)= & f_{q}\left(x_{0}-x+x-y\right)=-q^{2}\left(x_{0}-x\right) \\
& -q\left(x_{0}-x\right) L_{q}(x-y) \\
= & -q^{2}\left(x_{0}-x\right)+q\left(x_{0}-x\right) L(y-x) \\
\geq & -q^{2}\left(x_{0}-x\right)+q^{2}\left(x_{0}-x\right)=0 .
\end{aligned}
$$
$(2) \Longrightarrow(1)$. Since $f_{q} \in J_{q}\left(x-x_{0}\right)$, then 


$$
f_{q}\left(x-x_{0}\right)=q^{2}\left(x_{0}-x\right)=\left(q^{*}\left(f_{q}\right)\right)^{2} .
$$

Also, $f_{q}\left(x_{0}-y\right) \geq 0$, for every $y \in K$; therefore,

$$
\begin{aligned}
q^{2}\left(x_{0}-x\right) & =f_{q}\left(x-x_{0}\right)=f_{q}\left(x-y+y-x_{0}\right) \\
& \leq q(x-y) q^{*}\left(f_{q}\right)+f_{q}\left(y-x_{0}\right) \\
& \leq q(x-y) q^{*}\left(f_{q}\right)=q(x-y) q\left(x_{0}-x\right)
\end{aligned}
$$

for every $y \in K$; hence, we have

$$
q\left(x_{0}-x\right) \leq q(x-y)
$$

for all $y \in K$. This means that

$$
q\left(x_{0}-x\right)=\inf \{q(x-y): y \in K\} .
$$

Corollary 1. Let $Q$ be a family of seminorms on a locally convex space $E$ which determines the topology of $E, C$ a nonempty closed convex subset of $E, C_{0} \subseteq C$, and $P$ be a sunny $Q$-nonexpansive retraction of $C$ onto $C_{0}$. Let $J_{q}: E \longrightarrow E^{*}$ be single-valued duality mapping for each $q \in Q$; then,

$$
\left\langle x-P x, J_{q}(y-P x)\right\rangle \leq 0,
$$

for each $x \in C$ and $y \in C_{0}$.

Proof. Let $x \in C$ and $y \in C_{0}$. Set $x_{t}=P x+t(x-P x)$, for each $0 \leq t \leq 1$; then, $x_{t} \in C$ and

$$
q(P x-y)=q\left(P x_{t}-P y\right) \leq q\left(x_{t}-y\right),
$$

for each $q \in Q$. Now, from Theorem 9, there exists $f_{q} \in J_{q}(y-P x)$ such that $\left\langle P x-x_{t}, f_{q}\right\rangle=f_{q}\left(P x-x_{t}\right) \geq 0$. Since $J_{q}$ is single valued, we can say that

$$
\left\langle P x-x_{t}, J_{q}(y-P x)\right\rangle \geq 0,
$$

and since $P x-x_{t}=t(P x-x)$, then

$$
\left\langle x-P x, J_{q}(y-P x)\right\rangle \leq 0 \text {. }
$$

We now extend the result of Theorem 2.6.10 in [1] as follows.

Theorem 10. Let $E$ be a locally convex space and $J_{q}: E \longrightarrow E^{*}$ be a single-valued duality mapping. Then, $J_{q}$ is continuous from $\tau_{Q}$ to weak ${ }^{*}$ topology, for each $q \in Q$.

Proof. Let $q \in Q$ be fixed and arbitrary, $\left\{x_{\alpha}\right\}_{\alpha \in I} \subset E$, and $x \in E$ such that $x_{\alpha} \longrightarrow x$ in $\tau_{Q}$, and we prove that $J_{q} x_{\alpha} \longrightarrow J_{q} x$ in the weak $^{*}$ topology. First, assume that $q(x)=0$; then, $J_{q} x=0$ (from (13)). It is clear that $q^{*}\left(J_{q} x_{\alpha}\right)=q\left(x_{\alpha}\right)$, also $q \in Q$ is continuous Theorem 1.4 in [19]; therefore,

$$
\lim _{\alpha} q^{*}\left(J_{q} x_{\alpha}\right)=\lim _{\alpha} q\left(x_{\alpha}\right)=0
$$

and hence, $\lim _{\alpha}\left\langle y, J_{q} x_{\alpha}\right\rangle=0$, for every $y \in E$ that $q(y) \leq 1$ (from (9)). On the contrary, if $q(y)>1$, then $q(y / q(y))=1$; therefore, $\lim _{\alpha}\left\langle y / q(y), J_{q} x_{\alpha}\right\rangle=0$. So, we conclude that $\lim _{\alpha}\left\langle y, J_{q} x_{\alpha}\right\rangle=0$, for every $y \in E$. Hence, $J_{q} x_{\alpha} \longrightarrow 0$ in the weak $^{*}$ topology. Second, assume that $q(x) \neq 0$. Since

$$
J_{q} x_{\alpha}=\left\{j \in X^{*}:\left\langle x_{\alpha}, j\right\rangle=q\left(x_{\alpha}\right)^{2}=q^{*}(j)^{2}\right\},
$$

and $J_{q}$ is single-valued set $f_{\alpha}^{q}$ : $=J_{q} x_{\alpha}$. Then,

$$
\left\langle x_{\alpha}, f_{\alpha}^{q}\right\rangle=q\left(x_{\alpha}\right) q^{*}\left(f_{\alpha}^{q}\right), \quad q\left(x_{\alpha}\right)=q^{*}\left(f_{\alpha}^{q}\right) .
$$

Since $\left\{x_{\alpha}\right\}_{\alpha}$ is bounded with respect to $\tau_{Q}$, then there exists $N_{q}>0$ such that $q\left(x_{\alpha}\right) \leq N_{q}$, for each $\alpha \in I$. Now, it is shown that $\left\{f_{\alpha}^{q}\right\}_{\alpha \in I}$ is bounded in $E^{*}$.

One can see that $E^{*}$ is a locally convex space and the family of seminorms $\left\{p_{y}: y \in E\right\}$ defines the weak ${ }^{*}$ topology on it, where $p_{y}\left(x^{*}\right)=\left|\left\langle y, x^{*}\right\rangle\right|$, for each $x^{*} \in E$ (Chapter 5, Definition 1.1 in [22]). Hence,

$$
p_{y}\left(f_{\alpha}^{q}\right)=\left|\left\langle y, f_{\alpha}^{q}\right\rangle\right| \leq q(y) q^{*}\left(f_{\alpha}^{q}\right)=q(y) q\left(x_{\alpha}\right) \leq q(y) N_{q},
$$

when $q(y) \neq 0$. Also, if $q(y)=0$,

$$
\begin{aligned}
p_{y}\left(f_{\alpha}^{q}\right) & =\left|\left\langle y, f_{\alpha}^{q}\right\rangle\right| \leq \sup \left\{\left|f_{\alpha}^{q}(y)\right|: y \in E, q(y) \leq 1\right\} \\
& =q^{*}\left(f_{\alpha}^{q}\right)=q\left(x_{\alpha}\right) \leq N_{q},
\end{aligned}
$$

for each $\alpha \in I$. By applying (60) and (61), there exists $M_{q, y}>0$ related to each $y \in E$ and $q \in Q$, such that $p_{y}\left(f_{\alpha}^{q}\right) \leq M_{q, y}$, for each $\alpha \in I$. This means that $\left\{f_{\alpha}^{q}\right\}_{\alpha \in I}$ is bounded in the weak $^{*}$ topology of $E^{*}$. Putting $U_{q}=\{z \in E: q(z)<1\}$, we have $\left\{\left(1 / M_{q, y}\right) f_{\alpha}^{q}\right\}_{\alpha \in I} \subset U_{q}^{\circ}$, for each $y \in E$, where $U_{q}^{\circ}$ is the polar of $U_{q}$ and it is weak ${ }^{*}$ compact (Theorem 4). Then, from Theorem 5 , there exists a subnet $\left\{\left(1 / M_{q, y}\right) f_{\alpha_{\beta}}^{q}\right\}_{\beta_{0}}$ of $\left\{\left(1 / M_{q, y}\right) f_{\alpha}\right\}_{\alpha_{*}}$ such that $\left(1 / M_{q, y}\right) f_{\alpha_{\beta}}^{q} \longrightarrow f \in U_{q}^{\circ}$ in the weak $^{*}$ topology, equivalently

$$
\left\langle x, M_{q, y} f-f_{\alpha_{\beta}}^{q}\right\rangle \longrightarrow 0 .
$$

We know that the function $q^{*}$ on $E^{*}$ is lower semicontinuous in the weak $^{*}$ topology. Indeed, if $\left\{g_{\beta}\right\}_{\beta_{*}} \subset\left\{f \in E^{*}: q^{*}(f) \leq \alpha\right\}$ such that $g_{\beta} \longrightarrow g$ in the weak $^{*}$ topology, then

$$
\left|\left\langle y, g_{\beta}-g\right\rangle\right|=p_{y}\left(g_{\beta}-g\right) \longrightarrow 0,
$$

for each $y \in E$; therefore, $\left\langle y, g_{\beta}\right\rangle \longrightarrow\langle y, g\rangle$ and $q^{*}(g) \leq \alpha$, i.e.,

$$
g \in\left\{f \in E^{*}: q^{*}(f) \leq \alpha\right\}
$$

So, by applying Theorem $3, q^{*}$ is lower semicontinuous in the weak ${ }^{*}$ topology on $E^{*}$. Hence, we have

$$
q^{*}(f) \leq \liminf _{\beta} q^{*}\left(\frac{1}{M_{q, y}} f_{\alpha_{\beta}}^{q}\right)=\frac{1}{M_{q, y}} \liminf _{\beta} q\left(x_{\alpha_{\beta}}\right)=\frac{1}{M_{q, y}} q(x) .
$$
then

Since $x_{\alpha_{\beta}} \longrightarrow x$ in $\tau_{Q}$ and $x_{\alpha_{\beta}} \longrightarrow x$ in weak topology,

$$
\left\langle x-x_{\alpha_{\beta}}, f_{\alpha_{\beta}}^{q}\right\rangle \longrightarrow 0 .
$$

Therefore, using (62) and (66) implies that 


$$
\begin{aligned}
\left|\left\langle x, M_{q, y} f\right\rangle-q\left(x_{\alpha_{\beta}}\right)^{2}\right| & =\left|\left\langle x, M_{q, y} f\right\rangle-\left\langle x_{\alpha_{\beta}}, f_{\alpha_{\beta}}^{q}\right\rangle\right| \\
& \leq\left|\left\langle x, M_{q, y} f-f_{\alpha_{\beta}}^{q}\right\rangle\right|+\left|\left\langle x-x_{\alpha_{\beta}}, f_{\alpha_{\beta}}^{q}\right\rangle\right| \longrightarrow 0 .
\end{aligned}
$$

On the contrary, $q(x)^{2}\left(x_{\alpha_{\beta}}\right) \longrightarrow q(x)^{2}(x)$. Thus,

$$
\left\langle x, M_{q, y} f\right\rangle=q(x)^{2} .
$$

Since $q(x) \neq 0$, using (65), we have

$$
q(x)^{2}=\left\langle x, M_{q, y} f\right\rangle \leq q^{*}\left(M_{q, y} f\right) q(x) \leq q(x)^{2},
$$

and therefore,

$$
\begin{aligned}
\left\langle x, M_{q, y} f\right\rangle & =q(x)^{2}, \\
q(x) & =q^{*}\left(M_{q, y} f\right) .
\end{aligned}
$$

This means that $M_{q, y} f=J_{q} x$. Finally, since $f_{\alpha}^{q}=J_{q} x_{\alpha}$, from (62), we conclude that $J_{q} x_{\alpha} \longrightarrow J_{q} x$. This completes the proof.

In the next theorem, we prove an existence theorem of a sunny $Q$-nonexpansive retract.

Theorem 11. Let $Q$ be a family of seminorms on a real separated and complete locally convex space $E$ which determines the topology of $E, C$ a nonempty closed convex and bounded subset of $E$ such that every sequence in $C$ has a convergent subsequence, and $T: C \longrightarrow C$ be a Q-nonexpansive mapping such that Fix $(T) \neq \varnothing$, the fixed points set of $T$. Let $J_{q}: E \longrightarrow E^{*}$ be single valued for every $q \in Q$. Then, Fix $(T)$ is a sunny $Q$-nonexpansive retract of $C$ and the sunny Q-nonexpansive retraction of $C$ onto Fix $(T)$ is unique.

Proof. Let $x \in C$ be fixed; then, there exists a sequence $\left\{z_{n}\right\}$ in $C$ such that

$$
z_{n}=\frac{1}{n} x+\left(1-\frac{1}{n}\right) T z_{n}, \quad n \in \mathbb{N} .
$$

For this means, we define the function $K_{n}: C \longrightarrow C$, with

$$
K_{n}(t)=\frac{1}{n} x+\left(1-\frac{1}{n}\right) T(t),
$$

for each $n \in \mathbb{N}$ and $t \in C$. It is clear that $K_{n}$ is Q-nonexpansive because

$$
q\left(K_{n}\left(t_{1}\right)-K_{n}\left(t_{2}\right)\right) \leq\left(1-\frac{1}{n}\right) q\left(T\left(t_{1}\right)-T\left(t_{2}\right)\right) \leq q\left(t_{1}-t_{2}\right),
$$

for every $t_{1}, t_{2} \in C$. Then, by applying Theorem 6 , there exists a unique point $z_{n}$ in $C$ such that $K_{n}\left(z_{n}\right)=z_{n}$, for each $n \in \mathbb{N}$; therefore,

$$
z_{n}=\frac{1}{n} x+\left(1-\frac{1}{n}\right) T z_{n}, \quad n \in \mathbb{N} .
$$

Since $C$ is bounded in $\tau_{Q}$, there exists $M_{q}>0$ such that $q(y) \leq M_{q}$, for each $y \in C$ and $q \in Q$. Also,

$$
q\left(T z_{n}-z_{n}\right)=\frac{1}{n} q\left(T z_{n}-x\right) \leq \frac{2}{n} M_{q},
$$

and then,

$$
\lim _{n} q\left(T z_{n}-z_{n}\right)=0,
$$

for each $q \in Q$. Next, we show that the sequence $\left\{z_{n}\right\}$ converges to an element of $\operatorname{Fix}(T)$. In the other words, we show that the limit set of $\left\{z_{n}\right\}$ (denoted by $\mathfrak{S}\left\{z_{n}\right\}$ ) is a subset of Fix $(T)$. First, for each $z \in \operatorname{Fix}(T)$ and $n \in \mathbb{N}$, we have

$$
\left\langle z_{n}-x, J_{q}\left(z_{n}-z\right)\right\rangle \leq 0
$$

because $T z_{n}-x=n\left(T z_{n}-z_{n}\right)$ and

$$
\begin{aligned}
\left\langle T z_{n}-T z, J_{q}\left(z_{n}-z\right)\right\rangle & \leq q\left(T z_{n}-T z\right) q^{*}\left(J_{q}\left(z_{n}-z\right)\right) \\
& \leq q\left(z_{n}-z\right) q\left(z_{n}-z\right)=q^{2}\left(z_{n}-z\right) .
\end{aligned}
$$
have

Also, since $J_{q}$ is single valued, from definition (13), we

$$
\left\langle z-z_{n}, J_{q}\left(z_{n}-z\right)\right\rangle=-\left\langle z_{n}-z, J_{q}\left(z_{n}-z\right)\right\rangle=-q^{2}\left(z_{n}-z\right) .
$$

Therefore,

$$
\begin{aligned}
\left\langle z_{n}-x, J_{q}\left(z_{n}-z\right)\right\rangle= & \left\langle z_{n}-T z_{n}+T z_{n}-x, J_{q}\left(z_{n}-z\right)\right\rangle \\
= & \left\langle z_{n}-T z_{n}+n\left(T z_{n}-z_{n}\right), J_{q}\left(z_{n}-z\right)\right\rangle \\
= & (n-1)\left\langle T z_{n}-z_{n}, J_{q}\left(z_{n}-z\right)\right\rangle \\
= & (n-1)\left\langle T z_{n}-T z, J_{q}\left(z_{n}-z\right)\right\rangle \\
& +(n-1)\left\langle z-z_{n}, J_{q}\left(z_{n}-z\right)\right\rangle \\
\leq & (n-1)\left(q\left(T z_{n}-T z\right) q\left(z_{n}-z\right)-q\left(z_{n}-z\right)^{2}\right) \\
\leq & (n-1)\left(q\left(z_{n}-z\right)^{2}-q\left(z_{n}-z\right)^{2}\right)=0 .
\end{aligned}
$$

From our assumption, $\left\{z_{n}\right\}$ has a subsequence converges to a point in $C$. Let $\left\{z_{n_{i}}\right\}$ and $\left\{z_{n_{j}}\right\}$ be subsequences of $\left\{z_{n}\right\}$ such that $\left\{z_{n_{i}}\right\}$ and $\left\{z_{n_{j}}\right\}$ converge to $y$ and $z$, respectively. Then, (76) implies that

$$
\begin{aligned}
q(y-T y) & \leq q\left(y-z_{n_{i}}\right)+q\left(z_{n_{i}}-T z_{n_{i}}\right)+q\left(T z_{n_{i}}-T y\right) \\
& \leq 2 q\left(y-z_{n_{i}}\right)+q\left(z_{n_{i}}-T z_{n_{i}}\right) \longrightarrow 0,
\end{aligned}
$$

for each $q \in Q$. Since $E$ is separated, $T y=y$; hence, $y \in \operatorname{Fix}(T)$ and similarly $z \in \operatorname{Fix}(T)$. 
Now, $z_{n_{i}} \longrightarrow y$ and $z \in \operatorname{Fix}(T)$, and we claim that

$$
\left\langle y-x, J_{q}(y-z)\right\rangle=\lim _{i \longrightarrow \infty}\left\langle z_{n_{i}}-x, J_{q}\left(z_{n_{i}}-z\right)\right\rangle .
$$

Indeed, from the fact that $J_{q}$ is single valued and continuous from $\tau_{Q}$ to weak ${ }^{*}$ topology, we have

$$
\begin{aligned}
& \left|\left\langle z_{n_{i}}-x, J_{q}\left(z_{n_{i}}-z\right)\right\rangle-\left\langle y-x, J_{q}(y-z)\right\rangle\right| \\
& \quad=\left|\left\langle z_{n_{i}}-x, J_{q}\left(z_{n_{i}}-z\right)\right\rangle-\left\langle y-x, J_{q}\left(z_{n_{i}}-z\right)\right\rangle+\left\langle y-x, J_{q}\left(z_{n_{i}}-z\right)\right\rangle-\left\langle y-x, J_{q}(y-z)\right\rangle\right| \\
& \quad \leq\left|\left\langle z_{n_{i}}-y, J_{q}\left(z_{n_{i}}-z\right)\right\rangle\right|+\left|\left\langle y-x, J_{q}\left(z_{n_{i}}-z\right)-J_{q}(y-z)\right\rangle\right| \\
& \quad \leq q\left(z_{n_{i}}-y\right) q\left(z_{n_{i}}-z\right)+\left|\left\langle y-x, J_{q}\left(z_{n_{i}}-z\right)-J_{q}(y-z)\right\rangle\right| \\
& \quad \leq 2 q\left(z_{n_{i}}-y\right) M_{q}+\left|\left\langle y-x, J_{q}\left(z_{n_{i}}-z\right)-J_{q}(y-z)\right\rangle\right| \longrightarrow 0,
\end{aligned}
$$

and hence, by using (77),

$$
\left\langle y-x, J_{q}(y-z)\right\rangle=\lim _{i \longrightarrow \infty}\left\langle z_{n_{i}}-x, J_{q}\left(z_{n_{i}}-z\right)\right\rangle \leq 0 .
$$

Similarly,

$$
\left\langle z-x, J_{q}(z-y)\right\rangle \leq 0
$$

since

$J_{q}(y-z)=-J_{q}(z-y)$; then,

$$
\begin{aligned}
& q(y-z)^{2}=\left\langle y-z, J_{q}(y-z)\right\rangle \\
& \quad=\left\langle y-x, J_{q}(y-z)\right\rangle+\left\langle x-z, J_{q}(y-z)\right\rangle \\
& \quad=\left\langle y-x, J_{q}(y-z)\right\rangle+\left\langle z-x, J_{q}(z-y)\right\rangle \leq 0,
\end{aligned}
$$

that is, $q(y-z)=0$, for each $q \in Q$, and since $E$ is separated, $y=z$. Thus, $\left\{z_{n}\right\}$ converges to an element of $\operatorname{Fix}(T)$. Therefore, a mapping $P: C \longrightarrow C$ can be defined by $P x=\lim _{n} z_{n}$. Then, since $z_{n} \longrightarrow P x$, by using (84) and for each $z \in \operatorname{Fix}(T)$,

$$
\begin{aligned}
\left\langle x-P x, J_{q}(z-P x)\right\rangle & =\left\langle P x-x, J_{q}(P x-z)\right\rangle, \\
& =\lim _{n} \longrightarrow \infty\left\langle z_{n}-x, J_{q}\left(z_{n}-z\right)\right\rangle \leq 0 .
\end{aligned}
$$

It follows from Theorem 7 that $P$ is a sunny $Q$-nonexpansive retraction of $C$ onto $\operatorname{Fix}(T)$. Let $R$ be another sunny $Q$-nonexpansive retraction of $C$ onto $\operatorname{Fix}(T)$. Then, from Corollary 1 ,

$$
\left\langle x-R x, J_{q}(z-R x)\right\rangle \leq 0,
$$

for each $x \in C$ and $z \in \operatorname{Fix}(T)$. Putting $z=R x$ in (86) and $z=P x$ in (88), we have $\left\langle x-P x, J_{q}(R x-P x)\right\rangle \leq 0$ and $\left\langle x-R x, J_{q}(P x-R x)\right\rangle \leq 0$; then,

$$
q^{2}(R x-P x)=\left\langle R x-P x, J_{q}(R x-P x)\right\rangle \leq 0,
$$

for each $q \in Q$. Since $E$ is separated, $R x=P x$. This completes the proof.

Proposition 1. Let $Q$ be a family of seminorms on a separated locally convex space $E$ which determines the topology of E. Then,

$$
q(x)^{2}-q(y)^{2} \geq 2\langle x-y, j\rangle,
$$

for all $x, y \in E$ and $j \in J_{q} y$ such that $q(y) \neq 0$.

Proof. Let $j \in J_{q} x, x \in E$. Then,

$$
\begin{aligned}
& q(y)^{2}-q(x)^{2}-2\langle y-x, j\rangle \\
& \quad=q(x)^{2}+q(y)^{2}-2\langle y, j\rangle \\
& \quad \geq q(x)^{2}+q(y)^{2}-2 q(x) q(y) \\
& \quad \geq q(q(x)-q(y))^{2} \geq 0 .
\end{aligned}
$$

Theorem 12. Let $Q$ be a family of seminorms on a real separated and complete locally convex space $E$ which determines the topology of $E, C$ a nonempty closed convex and bounded subset of $E$ such that every sequence in $C$ has a convergent subsequence, and $T: C \longrightarrow C$ a $Q$-nonexpansive mapping such that Fix $(T) \neq \varnothing$. Let $J_{q}: E \longrightarrow E^{*}$ be single valued for each $q \in Q$ and $f$ be a $Q$-contraction on $C$; also, $\varepsilon_{n}$ is a sequence in $(0,1)$ such that $\lim _{n} \varepsilon_{n}=0$. Then, there exists a unique $x \in C$ and sunny $Q$-nonexpansive retraction $P$ of $C$ onto Fix $(T)$ such that the following sequence $\left\{z_{n}\right\}$, generated by

$$
z_{n}=\varepsilon_{n} f z_{n}+\left(1-\varepsilon_{n}\right) T z_{n}, \quad n \in \mathbb{N},
$$

converges to $P x$.

Proof. Since $f$ is a $Q$-contraction, there exists $0 \leq \beta<1$ such that

$$
q(f(x)-f(y)) \leq \beta q(x-y),
$$

for each $x, y \in E$ and $q \in Q$. We divide the proof into five steps.

Step 1: the existence of $z_{n}$ which satisfies (92).

This follows immediately from the fact that, for every $n \in \mathbb{N}$, the mapping $N_{n}: C \longrightarrow C$, given by

$$
N_{n} x:=\varepsilon_{n} f x+\left(1-\varepsilon_{n}\right) T x, \quad x \in C,
$$

is a $Q$-contraction. To see this, put $\beta_{n}=\left(1+\varepsilon_{n}(\beta-1)\right)$, $0 \leq \beta_{n}<1, n \in \mathbb{N}$. Then, we have 


$$
\begin{aligned}
q\left(N_{n} x-N_{n} y\right) & \leq \varepsilon_{n} q(f x-f y)+\left(1-\varepsilon_{n}\right) q(T x-T y) \\
& \leq \varepsilon_{n} \beta q(x-y)+\left(1-\varepsilon_{n}\right) q(x-y) \\
& =\left(1+\varepsilon_{n}(\beta-1)\right) q(x-y) \\
& =\beta_{n} q(x-y)<q(x-y) .
\end{aligned}
$$

Therefore, by Theorem 6 , there exists a unique point $z_{n} \in C$ such that $N_{n} z_{n}=z_{n}$, that is,

$$
z_{n}=\varepsilon_{n} f z_{n}+\left(1-\varepsilon_{n}\right) T z_{n} .
$$

Step 2: $\lim _{n} q\left(T z_{n}-z_{n}\right)=0$, for each $q \in Q$.

Since $C$ is bounded in $\tau_{Q}$, there exists $M_{q}>0$ such that $q(y) \leq M_{q}$, for each $y \in C$ and $q \in Q$.

Then,

$$
\begin{aligned}
q\left(T z_{n}-z_{n}\right) & =\varepsilon_{n} q\left(T z_{n}-f z_{n}\right) \leq 2 M_{q} \varepsilon_{n}, \\
\lim _{n} q\left(T z_{n}-z_{n}\right) & =0,
\end{aligned}
$$

for each $q \in Q$.

Step 3: $\mathfrak{S}\left(\left\{z_{n}\right\}\right) \subset \operatorname{Fix}(T)$, where $\mathfrak{S}\left(\left\{z_{n}\right\}\right)$ denotes the set of $\tau_{\mathrm{Q}}$-limit points of subsequences of $\left\{z_{n}\right\}$.

Let $z \in \mathbb{S}\left(\left\{z_{n}\right\}\right)$ and $\left\{z_{n_{k}}\right\}$ be a subsequence of $\left\{z_{n}\right\}$ such that $z_{n_{k}} \longrightarrow z$. By using (97), we have

$$
\begin{aligned}
q(T z-z) & \leq q\left(T z-T z_{n_{k}}\right)+q\left(T z_{n_{k}}-z_{n_{k}}\right)+q\left(z_{n_{k}}-z\right) \\
& \leq 2 q\left(z_{n_{k}}-z\right)+q\left(T z_{n_{k}}-z_{n_{k}}\right) \longrightarrow 0,
\end{aligned}
$$

for each $q \in Q$. Hence, $q(T z-z)=0$, for each $q \in Q$, and since $E$ is separated, we conclude that $z \in \operatorname{Fix}(T)$.

Step 4: there exists a unique sunny $Q$-nonexpansive retraction $P$ of $C$ onto $\operatorname{Fix}(T)$ and $x \in C$ such that

$$
K:=\underset{n}{\limsup }\left\langle x-P x, J_{q}\left(z_{n}-P x\right)\right\rangle \leq 0 .
$$

We know from Theorem 10 that $J_{q}$ is continuous from $\tau_{Q}$ to weak ${ }^{*}$ topology; then, by Theorem 11 , there exists a unique sunny $Q$-nonexpansive retraction $P$ of $C$ onto $\operatorname{Fix}(T)$. Theorem 6 guarantees that $f P$ has a unique fixed point $x \in C$. Since

$$
K=\limsup _{n}\left\langle x-P x, J_{q}\left(z_{n}-P x\right)\right\rangle,
$$

by the definition of limsup, there exists subsequence $\left\{z_{n_{i}}\right\}$ of $\left\{z_{n}\right\}$ such that

$$
K=\lim _{i}\left\langle x-P x, J_{q}\left(z_{n_{i}}-P x\right)\right\rangle .
$$

On the contrary, every sequence in $C$ has a convergent subsequence; then, there exists subsequence $\left\{z_{n_{i_{k}}}\right\}$ of $\left\{z_{n_{i}}\right\}$ such that $z_{n_{i_{k}}} \longrightarrow z$ and $z \in C$.

By Step 3, we have $z \in \operatorname{Fix}(T)$. From Theorem 7, we have

$$
\left\langle x-P x, J_{q}(z-P x)\right\rangle \leq 0,
$$

and since $J_{q}$ is continuous, then

$$
\lim _{k}\left\langle x-P x, J_{q}\left(z_{n_{i_{k}}}-P x\right)\right\rangle=\left\langle x-P x, J_{q}(z-P x)\right\rangle .
$$

Therefore, by applying (101)-(103), we have $K \leq 0$.

Step 5: $\left\{z_{n}\right\}$ converges to $P x$ in $\tau_{Q}$.

Since $f P x=x$, thus $(f-I) P x=x-P x$. Now, from Proposition 1 and our assumption, we have

$$
\begin{aligned}
\varepsilon_{n}( & \beta-1) q\left(z_{n}-P x\right)^{2} \\
\geq & {\left[\varepsilon_{n} \beta q\left(z_{n}-P x\right)+\left(1-\varepsilon_{n}\right) q\left(z_{n}-P x\right)\right]^{2}-q\left(z_{n}-P x\right)^{2} } \\
\geq & {\left[\varepsilon_{n} q\left(f z_{n}-f(P x)\right)+\left(1-\varepsilon_{n}\right) q\left(T z_{n}-P x\right)\right]^{2}-q\left(z_{n}-P x\right)^{2} } \\
\geq & 2\left\langle\varepsilon_{n}\left(f z_{n}-f(P x)\right)+\left(1-\varepsilon_{n}\right)\left(T z_{n}-P x\right)\right. \\
& \left.-\left(z_{n}-P x\right), J_{q}\left(z_{n}-P x\right)\right\rangle \\
= & -2 \varepsilon_{n}\left\langle(f-I) P x, J_{q}\left(z_{n}-P x\right)\right\rangle \\
= & -2 \varepsilon_{n}\left\langle x-P x, J_{q}\left(z_{n}-P x\right)\right\rangle,
\end{aligned}
$$

for each $n \in \mathbb{N}$. Hence,

$$
q\left(z_{n}-P x\right)^{2} \leq \frac{2}{1-\beta}\left\langle x-P x, J_{q}\left(z_{n}-P x\right)\right\rangle,
$$

for each $q \in Q$. Since $P x \in \operatorname{Fix}(T)$, from (99) and (105), we conclude that

$$
\underset{n}{\limsup } q\left(z_{n}-P x\right)^{2} \leq \frac{2}{1-\beta} \limsup _{n}\left\langle x-P x, J_{q}\left(z_{n}-P x\right)\right\rangle \leq 0,
$$

for each $q \in Q$. That is, $z_{n} \longrightarrow P x$ in $\tau_{Q}$.

\section{Numerical Example}

The following examples illustrate Theorem 12. The first example is in the setting of a locally convex space that is not normable and the rest in finite dimensional spaces.

Example 1. Let $\varepsilon$ be an arbitrary positive number, $E=C([0,1])$, the set of all continuous complex valued functions, and $\varepsilon_{n}=(1 / n)$. For each $x \in[0,1]$, the seminorm $q$ on $E$ is defined by $q_{x}(f)=|f(x)|$, for each $f \in E$. Note that $E$ is a locally convex space with the topology induced by Q. It can be proved by Urysohn's Lemma that it is not normable.

In Theorem 12, let $C=N\left(x_{0}, \varepsilon, 0\right)=\left\{f: q_{x_{0}}(f)\right.$ $\left.=\left|f\left(x_{0}\right)\right|<\varepsilon\right\}$ be convex neighborhood of 0 with $x_{0} \in[0,1]$ and $\left|x_{0}\right|<(\varepsilon / 2)$. Suppose that $I$ is the identity mapping on the complex numbers, and let $T, F: E \longrightarrow E$ be defined by $T(f)$. The following examples illustrate Theorem 12. The first example is in the setting of a locally convex space that is not normable and the rest in finite dimensional spaces. 
Example 2. Let $\varepsilon$ be an arbitrary positive number and $E=$ $C([0,1])$ be the set of all continuous complex valued functions and $\varepsilon_{n}=(1 / n)$. For each $x \in[0,1]$, we define the seminorm $q_{x}$ on $E$ by $q_{x}(f)=|f(x)|$, for each $f \in C_{C}([0,1])$. Note that $E$ is a locally convex space with the topology induced by $Q$. It can be proved by Urysohn's Lemma which is not normable.

In Theorem 12, let $C=N\left(x_{0}, \varepsilon, 0\right)=\left\{f: q_{x_{0}}\right.$ $\left.(f)=\left|f\left(x_{0}\right)\right|<\varepsilon\right\}$ be convex neighborhood of 0 with $x_{0} \in[0,1]$ and $\left|x_{0}\right|<(\varepsilon / 2)$. Suppose that $I$ is the identity mapping on the complex numbers, and let $T, F: E \longrightarrow E$ be defined by $T(f)=(1 / 2)(f+I)$ and $F(f)=(1 / 2) f$, respectively. It is obvious that $T$ is $Q$-nonexpansive and $F$ is $Q$-contraction mapping on $C$. It can be verified that $P(f)=$ $I$ is a sunny $Q$-nonexpansive retraction from $C$ onto $\operatorname{Fix}(T)=\{I\}$; then, obviously, we have the sequence $\left\{f_{n}\right\}_{n \in \mathbb{N}}=\{(1-(1 / n)) I\}_{n \in \mathbb{N}}$ generated by (92) which converges to $P I=I$ with respect to the topology induced by $Q$.

Example 3. In Theorem 12 , let $E=\mathbb{R}, \varepsilon_{n}=(1 / n)$, and $q(x)=|x|$ be the only seminorm on $\mathbb{R}$, i.e., $Q=\{||$.$\} . Let$ $C=[0,2]$ and $T(x)=(1 / 2) x+1$ be a $Q$-nonexpansive mapping and $f(x)=(1 / 2) x$ be a $Q$-contraction mapping on $[0,2]$. It is obvious $P(x)=2$ is a sunny $Q$-nonexpansive retraction from $[0,2]$ onto $\operatorname{Fix}(T)=\{2\}$ and the sequence $\left\{z_{n}\right\}_{n \in \mathbb{N}}=\{2-(2 / n)\}_{n \in \mathbb{N}}$ generated by $(92)$ converges to $P 1=$ 2 and $F(f)=(1 / 2) f$, respectively. It is obvious that $T$ is $Q$-nonexpansive and $F$ is $Q$-contraction mapping on $C$. It can be verified that $P(f)=I$ is a sunny $Q$-nonexpansive retraction from $C$ onto $\operatorname{Fix}(T)=\{I\}$; then, obviously, we have the sequence $\left\{f_{n}\right\}_{n \in \mathbb{N}}=\{(1-(1 / n)) I\}_{n \in \mathbb{N}}$ generated by (92) converges to $P I=I$ with respect to the topology induced by $Q$.

Example 4. In Theorem 12 , let $E=\mathbb{R}, \varepsilon_{n}=(1 / n)$, and $q(x)=|x|$ be the only seminorm on $\mathbb{R}$, i.e., $Q=\{||$.$\} . Let$ $C=[0,2]$ and $T(x)=(1 / 2) x+1$ be a $Q$-nonexpansive mapping and $f(x)=(1 / 2) x$ be a $Q$-contraction mapping on $[0,2]$. It is obvious $P(x)=2$ is a sunny $Q$-nonexpansive retraction from $[0,2]$ onto $\operatorname{Fix}(T)=\{2\}$ and the sequence $\left\{z_{n}\right\}_{n \in \mathbb{N}}=\{2-(2 / n)\}_{n \in \mathbb{N}}$, generated by (92), converges to $P 1=2$.

Example 5. In Theorem 12, assume $E=\mathbb{R}^{2}$ and $\varepsilon_{n}=(1 / n)$, and $q_{1}(x, y)=|x|$ and $q_{2}(x, y)=|y|$ are two seminorms on $\mathbb{R}^{2}$, i.e., $Q=\left\{q_{1}, q_{2}\right\}$. Let $C=[0,2] \times[0,2]$ and $T(x, y)=$ $((1 / 2) x+1,(1 / 3) y+1)$ be a $Q$-nonexpansive mapping and $f(x, y)=((1 / 2) x,(1 / 3) y)$ be a $Q$-contraction mapping on $[0,2] \times[0,2]$ in Theorem 11 . It is easy to check that $P(x, y)=(2,(3 / 2))$ is a sunny $Q$-nonexpansive retraction from $[0,2] \times[0,2]$ onto $\operatorname{Fix}(T)=\{(2,(3 / 2))\}$, and the sequence $\left\{z_{n}\right\}_{n \in \mathbb{N}}=\left\{\left(x_{n}, y_{n}\right)\right\}_{n \in \mathbb{N}}=\{(2-(2 / n),(3 / 2)-(3$ $/ 2 n))\}_{n \in \mathbb{N}}$ generated by (92) converges to $P(1,1)=(2,(3 / 2))$.

\section{Data Availability}

The data used to support the findings of the study are available within the article.

\section{Conflicts of Interest}

The authors declare that they have no conflicts of interest.

\section{Acknowledgments}

This work was supported by the National Natural Science Foundation of China (no. 11671365).

\section{References}

[1] R. P. Agarwal, D. Oregan, and D. R. Sahu, "Fixed point theory for Lipschitzian-type mappings with applications," in Topological Fixed Point Theory and its ApplicationsSpringer, Berlin, Germany, 2009.

[2] A. Bnouhachem, "A descent SQP alternating direction method for minimizing the sum of three convex functions," Journal of Nonlinear Variational Analysis, vol. 4, pp. 469-482, 2020.

[3] P. Cubiotti and J.-C. Yao, "On the Cauchy problem for a class of differential inclusions with applications," Applicable Analysis, vol. 99, no. 14, pp. 2543-2554, 2020.

[4] T. H. Cuong, J.-C. Yao, and N. D. Yen, "Qualitative properties of the minimum sum-of-squares clustering problem," Optimization, vol. 69, no. 9, pp. 2131-2154, 2020.

[5] A. Moudafi and N. Lehdili, "From progressive decoupling of linkages in variational inequalities to fixed-point problems," Applied Set-Valued Analysis and Optimization, vol. 2, pp. 159-173, 2020.

[6] M. Tian and G. Xu, "Inertial modified Tseng's extragradient algorithms for solving monotone variational inequalities and fixed point problems," Journal of Nonlinear Functional Analysis, vol. 2020, Article ID 35, 2020.

[7] S. Cho, "A monotone Bregan projection algorithm for fixed point and equilibrium problems in a reflexive Banach space," Filomat, vol. 34, no. 5, pp. 1487-1497, 2020.

[8] S. Y. Cho, "A convergence theorem for generalized mixed equilibrium problems and multivalued asymptotically nonexpansive mappings," Journal of Nonlinear Convex Analysis, vol. 21, pp. 1017-1026, 2020.

[9] X. Qin, L. Wang, and J. C. Yao, "Inertial splitting method for maximal monotone mappings," Journal of Nonlinear Convex Analysis, vol. 21, pp. 2325-2333, 2020.

[10] Y. Shehu, X. Qin, and J. C. Yao, "Weak and linear convergence of proximal point algorithm with reflections," Journal of Nonlinear Convex Analysis, vol. 22, pp. 299-307, 2021.

[11] Y. Shehu and J. C. Yao, "Rate of convergence for inertial iterative method for countable family of certain quasi-nonexpansive mappings," Journal of Nonlinear Convex Analysis, vol. 21, pp. 533-541, 2020.

[12] S. Dhompongsa, P. Kumam, and E. Soori, "Fixed point properties and $\$ \$\{\mid$ varvec $\{\mathrm{Q}\}\} \$ \$ \mathrm{Q}$-nonexpansive retractions in locally convex spaces," Results in Mathematics, vol. 73, no. 2, 2018.

[13] P. Chuasuk, A. Farajzadeh, and A. Kaewcharoen, "An iterative algorithm for solving split feasibility problems and fixed point problems in p-uniformly convex and smooth Banach spaces," Journal of Computational Analysis and Applications, vol. 28, no. 1, pp. 49-66, 2020.

[14] P. Lohawech, A. Kaewcharoen, and A. Farajzadeh, "Algorithms for the common solution of the split variational inequality problems and fixed point problems with applications," Journal of Inequalities and Applications, vol. 358, pp. 1-17, 2018. 
[15] P. Chuadchawna, A. Farajzadeh, and A. Kaewcharoen, "On convergence theorems for two generalized nonexpansive multivalued mappings in hyperbolic spaces," Thai Journal of Mathematics, vol. 17, no. 2, pp. 445-461, 2019.

[16] P. Chuasuk, A. Farajzadeh, A. Farajzadeh, A. Kaewcharoen, and R. P. Agarwal, "An iterative process for a hybrid pair of a Bregman strongly nonexpansive single-valued mapping and a finite family of Bregman relative nonexpansive multi-valued mappings in Banach spaces," Carpathian Journal of Mathematics, vol. 33, no. 3, pp. 287-300, 2017.

[17] N. Hussain, M. Lashkarizadeh Bami, and E. Soori, "An implicit method for finding a common fixed point of a representation of nonexpansive mappings in Banach spaces," Fixed Point Theory and Applications, vol. 2014, no. 1, Article ID 238, 2014.

[18] W. Takahashi, Nonlinear Functional Analysis: Fixed Point Theory and its Applications, Yokohama Publishers, Yokohama, Japan, 2000.

[19] V. Barbu, Convexity and Optimization in Banach Spaces, Springer, Berlin, Germany, 2012.

[20] M. S. Osborne, Locally Convex Spaces, Springer, Switzerland, 2014.

[21] V. Runde, A Taste of Topology, Springer, Berlin, Germany, 2005.

[22] J. B. Conway, A Course in Functional Analysis, Springer, Berlin, Germany, 1990. 